\title{
Detailed Clinician Updates are Critical to Educational Success for Pediatric Patients With ME/CFS
}

Received: October 17, 2017; Accepted: March 25, 2018; Published: March 31, 2018

\section{Commentary}

Clinicians treating pediatric patients for ME/CFS are becoming increasingly aware of the critical role they play in assisting patients and their families in accessing special services that allow them to be successful in school. They understand that detailed physician letters, which include a diagnosis; a prognosis; lists of symptoms and medication side effects; and recommended accommodations and modifications to the school environment, will be more far effective in convincing a review committee to designate the student as "Other Health Impaired" (OHI) than a generic diagnosis form [1].

Educators providing special services are much better prepared to cope with other diseases and conditions that do not have the variability of ME/CFS. Specific learning disabilities, Autism, Attention Deficit Hyperactivity disorder, or visual impairments may involve symptomatic changes, but these usually have some predictability or at least fall within a known progression. ME/CFS, at least from the perspective of educators, does not [2].

Students suffering from ME/CFS can manifest widely-varying symptoms and degrees of symptoms that fluctuate without warning. A child capable of attending school successfully on a full or abbreviated schedule for two or three weeks may unexpectedly experience extreme post-exertional malaise (a "crash") lasting days or weeks, preventing attendance or even the completion of school assignments at home. Parents will not normally seek out their clinicians for appointments unless such a malaise extends significantly beyond those previously experienced [3].

School officials will often have little context for such prolonged absences (especially during a first or second occurrence) and may demand additional medical documentation to forgive work, extend deadlines, or approve homebound tutoring services. To the clinician, such events are an expected consequence of the disease, and often no reason is seen for a formal update to the physician's letter on file with the school.

A similar scenario may play out when the physician recommends
Faith Newton*

ME/CFS Education Initiative, Delaware State University, DuPont Highway, Delaware, USA

\author{
Corresponding author:
}

Dr. Faith Newton

fnewton@desu.edu

ME/CFS Education Initiative, Delaware State University, 1200 N. DuPont Highway, DE 19901, USA.

Tel: $302-229-7198$

Citation: Newton F (2018) Detailed Clinician Updates are Critical to Educational Success for Pediatric Patients With ME/CFS. Transl Biomed. Vol.9 No.1:143

a new medication or treatment. Often (as with Beta-blockers) the physician will try medications in a "trial and error" sequence, warning the parents that six or eight weeks might be required to see improvements; that the initial response might actually include a worsening of symptoms; or that this sequence might have to be repeated several times to determine which medication will be most effective.

If the physician letter is not updated in detail at such times, the parents and the child may be placed in a difficult position with the school. Often clinicians will depend on the parents' knowledge of the disease to relay their detailed comments about potential changes during the trial period, or with regard to a lengthy "crash." The parents may well be quite articulate and quite competent to relay this information accurately, but field experience shows that many school districts will discount this information if not accompanied by a detailed physician's update. As a result, services may be refused, credits may be lost, and the student's educational progress may be endangered.

The most effective mechanism to ensure the continuation of a smoothly functioning partnership between these families and the schools is for physicians to invest the time to complete a detailed clinical update on every occasion that they foresee the possibility of significant (even if temporary) changes in function. 


\section{References}

1 Newton F (2017) Chronic fatigue syndrome. National Association of special education teachers www.naset.org/3349.0.html.

2 Newton F (2015) Improving academic success for students with myalgic encephalomyelitis/chronic fatigue syndrome. Fatigue: Biomedicine, Health and Behavior 3: 97-103.

3 Rowe PC, Underhill RA, Friedman KJ, Gurwitt A, Medow MS, et al. (2017) Myalgic encephalomyelitis/chronic fatigue syndrome diagnosis and management in young people: A Primer. Frontiers in Pediatrics. 